\title{
Intersektionalität in der Internetforschung
}

\author{
Tanja Carstensen / Gabriele Winker
}

\begin{abstract}
Das Konzept der Intersektionalität entwickelt sich in der Geschlechterforschung zurzeit zu einem neuen Paradigma. Intersektionalität ist der Versuch, die Verwobenheiten und Wechselwirkungen von verschiedenen Ungleichbeitskategorien bzw. Herrschaftsverbältnissen zu analysieren. Auch die sozialwissenschaftliche Internetforschung behandelt seit Jabren Fragen der (Re-)Produktion von sozialen Ungleichbeiten und Diskriminierungsmechanismen. Allerdings sind die Fragestellungen heterogen. Im vorliegenden Artikel geben wir zunächst einen Überblick über den sozialwissenschaftlichen Forschungsstand zum Verbältnis von Internet und sozialer Ungleichbeit. Anschließend stellen wir das Konzept der intersektionalen Mebrebenenanalyse vor. Im Mittelpunkt des Textes stebt der Vorschlag für ein Forschungsdesign, anhand dessen intersektionale Ansprüche innerhalb der sozialwissenschaftlichen Internetforschung verwirklicht werden und einige der momentan unverbundenen Forschungsansätze zu Ungleichbeit und Internet zusammengefübrt werden können.
\end{abstract}

Schlagwörter: Geschlechterforschung, soziale Ungleichheit, Intersektionalität, Mehrebenenanalyse, Internet

In der Geschlechterforschung avanciert gegenwärtig das Konzept der Intersektionalität zu einem neuen Paradigma. Es bezeichnet die Verwobenheiten und Wechselwirkungen von verschiedenen Differenzkategorien bzw. Herrschaftsverhältnissen. Zwar ist in der Frauen- und Geschlechterforschung seit Jahrzehnten unbestritten, dass neben Geschlecht auch andere Kategorien wie race, Klasse, Bildung oder Alter bei der Analyse von Macht- und Herrschaftsverhältnissen Berücksichtigung finden sollten (Carby 1982, Combahee River Collective 1982, Crenshaw 1989, Collins 1993, Brah/Phoenix 2004, Yuval-Davis 2006). Die Frage, wie diese Kategorien bzw. die entsprechenden Herrschaftsverhältnisse Rassismus, Klassismus, Heteronormativismus oder Sexismus miteinander verwoben sind und zusammenwirken, wird aber erst seit einiger Zeit systematisch behandelt (u. a. Knapp 2005, Davis 2008, Winker/Degele 2009).

Auch in der sozialwissenschaftlichen Internetforschung haben Fragen nach der (Re-)Produktion und (De-)Stabilisierung sozialer Ungleichheit von Beginn an einen zentralen Stellenwert eingenommen und sind damit für intersektionale Überlegungen anschlussfähig. Gleichzeitig bleiben die Fragstellungen so divers wie das Internet selbst und setzen auf unterschiedlichsten Ebenen an.

In diesem Artikel möchten wir den Versuch unternehmen, die intersektionale Mehrebenenanalyse, wie sie Winker/Degele (2009) entwickelt haben, auf das Feld der sozialwissenschaftlichen Internetforschung anzuwenden. Hierfür geben wir zunächst einen Überblick über den sozialwissenschaftlichen Forschungsstand zum Verhältnis von Internet und sozialer Ungleichheit. Anschließend wird das Konzept der intersektionalen Mehrebenenanalyse vorgestellt und seine methodische Vorgehensweise skizziert. Hiervon ausgehend stellen wir ein Forschungsdesign vor, anhand dessen intersektionale Ansprüche innerhalb der sozialwissenschaftlichen Internetforschung verwirklicht werden können, und illustrieren das vorgeschlagene Vorgehen an einem Fallbeispiel. Ziel ist, einige der verschiedenen, momentan unverbundenen Forschungsansätze zu Ungleichheit und Internet zusammenzuführen und dadurch auch die Zusammenhänge zwischen den heterogenen Befunden herauszuarbeiten. 


\section{Internetforschung: Thema soziale Ungleichheit}

Bereits in der Anfangszeit des Internets in den 1990er Jahren, als in der Öffentlichkeit, aber auch in den Sozialwissenschaften, vor allem erste Hoffnungen und Befürchtungen hinsichtlich der Folgen des Internets für Individuum und Gesellschaft thematisiert wurden, waren Fragen nach Teilhabe und Partizipation bzw. nach sozialer Spaltung zentral (Rheingold 1994, Kleinsteuber 1995, Barlow 1996, Bühl 1998, Leggewie/Maar 1998). Diese frühen Prognosen waren meist von technikdeterministischen Deutungsmustern der neuen Technologie geprägt, es dominierte die Frage nach den - positiven oder negativen - Auswirkungen des Internets, wodurch Technik einseitige Wirkungsmacht zugeschrieben und ihre soziale Konstruiertheit und damit auch Gestaltbarkeit ausgeblendet wurde (vgl. Carstensen 2007, Münker 2009).

Mittlerweile ist die sozialwissenschaftliche Internetforschung sehr ausdifferenziert. Fragen nach Macht- und Herrschaftsverhältnissen sind dabei nach wie vor zentraler Bestandteil. Der Forschungsstand lässt sich entlang vier zentraler Forschungslinien differenzieren: Zugang, Nutzung und Konstruktion des Internets sowie die Beschaffenheit des „Cyberspace“, die im Folgenden vorgestellt werden.

\subsection{Zugang zum Internet: digital divide}

Fragen des ungleichen Zugangs zum Internet wurden und werden insbesondere unter dem Schlagwort digital divide thematisiert. Durch das Internet entsteht, so die These, eine gesellschaftliche Spaltung, die soziale Ungleichheit und Ausgrenzung nicht abbaut, sondern vertieft und damit eine neue Differenzierungslinie zwischen „information haves“ und „information have nots“ etabliert (u. a. Weizenbaum 1999, Bühl 1998: 355f., Norris 2001).

Inzwischen liegt eine Reihe von differenzierten empirischen Ergebnissen vor, die darlegen, wovon der Zugang zum Internet abhängt. Alter, Bildung und Einkommen sind dabei zentrale Kategorien, aber auch diverse andere werden behandelt. So widmen sich Untersuchungen (genauso wie politische Initiativen) zum digital divide beispielsweise den Möglichkeiten und Grenzen der gesellschaftlichen Teilhabe über das Internet von Personen mit Behinderungen (z. B. Dobransky/Hargittai 2006), Senior(inn)en (z. B. Fuglsang 2005), sozial benachteiligten und bildungsschwachen Jugendlichen (z. B. Iske/ Klein/Kutscher 2004) oder den Offliner(inne)n mit älteren Frauen als Kerngruppe (Gerhards/Mende 2009). Weitere Studien untersuchen die Reichweite digitaler Ungleichheit in Ostasien (Ono 2006), die Bedeutung von Bildung bzw. sozialem und kulturellem Kapital (Zillien 2006), von Gender (Winker 2004, Wasserman/Richmond-Abbott 2005), Stadt-Land-Unterschieden (Jäckel/Lenz/Zillien 2005) sowie der Kategorie race (z. B. Fairlie 2004) und des digital divide aus globaler Perspektive (z. B. Norris 2001).

Einen sehr guten Überblick darüber, wie sich die Zugangszahlen in der Bundesrepublik quantitativ entwickeln, bieten jährlich der (N)Onliner-Atlas (Initiative D21 2010) und die ARD/ZDF-Onlinestudien (z. B. van Eimeren/Frees 2011). Hier wird durch differenziertes Vorgehen und insbesondere durch die Kombination mehrerer Ungleichheitskategorien bei der Analyse deutlich, wie groß die digitale Kluft wird, werden beispielsweise Geschlecht und Alter zusammen untersucht. Zwar sind 78,2 \% der Männer und 68,5\% der Frauen im Internet, und in der Gruppe der 14 bis 19-jährigen sogar 98,9\% der Mädchen und 98,5 \% der Jungen. Gravierend wird die Kluft hingegen bei den über 70-jährigen; hier sind es nur 9,1 \% Frauen, aber immerhin 29,8 \% Männer (ebd.: 337). Diese überwiegend quantitativen Daten liefern bereits Hinweise darauf, dass sich verschiedene Ungleichheitskategorien gegenseitig verstärken oder abschwächen kön- 
nen. Für die genauere Betrachtung der Verwobenheiten verschiedener Kategorien bedarf es weiterführender qualitativer Analysen.

\subsection{Unterschiedliche Nutzung des Internets: (De-)Stabilisierung sozialer Ungleichbeit}

Auch hinsichtlich unterschiedlicher Nutzungsweisen waren Fragen nach sozialer Ungleichheit von Beginn an zentral (vgl. u. a. Schönberger 1999, Krotz 2001, Consalvo/ Paasonen 2002, Münker/Roesler 2002, Wellman/Haythornthwaite 2002). Zum einen sind dadurch eine Vielzahl von Nutzungstypologien entstanden, die aufzeigen, welche Gruppen das Internet wie nutzen, welche Nutzungsmuster sich herausbilden (z. B. Emmer/Füting/Vowe 2006, für das Web 2.0 auch: Mocigemba 2006, Schmidt 2006, Lenhart/ Madden 2007, Gerhards/Klinger/Trump 2008, Busemann/Gscheidle 2010) und welche Effekte diese auf soziale Ungleichheit und Teilhabe haben. Hepp/Suna/Welling (2009) verdeutlichen beispielsweise die Integrations- und Segregationspotenziale von Migrant(inn)en durch Nutzung des Internets (auch: Hunger/Kissau 2009, Hepp 2009). Ähnlich fragt auch Henning (2008) nach der sozial-integrativen Rolle ethnisch orientierter Online-Foren für Jugendliche mit türkischem Migrationshintergrund. Carstensen/Winker (2005a) zeigen am Beispiel der Internetnutzung von Frauenprojekten, wie subalterne Gegenöffentlichkeiten gestärkt werden können. Groß/Carstensen/Winker (2008) untersuchen Möglichkeiten und Grenzen gesellschaftlicher Teilhabe am Beispiel der Internetnutzung von Erwerbslosen. Hesse (2008) untersucht die Möglichkeiten für Beteiligung an Öffentlichkeit in der Blogosphäre unter Geschlechterperspektiven. Und Ahrens (2009) arbeitet an alltäglichen Internetpraktiken in Beziehungen heraus, wie die Internetnutzung mit der Herstellung von Geschlechterrollen verknüpft ist und inwiefern es bei der Integration des Internets in den Alltag zu Gender-Demokratisierungsprozessen kommt.

Zum anderen gibt es diverse Ansätze, die unterschiedliche Nutzungsweisen konzeptualisieren und versuchen, das „Wie“ der Nutzung zu erforschen (DiMaggio/Hargittai 2001, Mossberger/Tolbert/Stansbury 2003). Die digitale Spaltung der Gesellschaft verläuft hiernach nicht allein zwischen Besitz und Nichtbesitz von Computer und Internetzugang, sondern auch entlang der Nutzungspraktiken. In diesem Zusammenhang werden in der Regel Medienkompetenz, technische Ausstattung, materielle Ressourcen, Interesse und Motivation als wichtige Einflussfaktoren genannt (Hargittai 2002, Iske et al. 2004), die unterschiedliche Nutzungspraxen, Identitäten und damit ungleiche gesellschaftliche Beteiligungschancen konstituieren. Zillien (2006) spricht von „Wissenskluft", um zu verdeutlichen, dass Anwender(innen) des Internets aus ihrem Nutzungsverhalten unterschiedliche Erträge ziehen, weil sie unterschiedliche Voraussetzungen wie Nutzungsmotive und Kompetenzen mitbringen, die durch ihre jeweilige Schichtzugehörigkeit beeinflusst werden. Auch Marr (2005: 232) weist darauf hin, dass eine Konzentration auf strukturelle Benachteiligungen zu kurz greife. Vielmehr müssten auch die mentalen und motivationalen Barrieren, der fehlende bzw. sich nicht erschließende Nutzen des Internets und das daraus resultierende „Nicht-Wollen“ vieler Offliner(innen) stärker untersucht werden. DiMaggio/Hargittai (2001) verweisen darüber hinaus auf die Bedeutung der Nutzungsautonomie in Bezug auf Ort und Zeit sowie auf die Bedeutung sozialer Unterstützungsstrukturen; und Winker (2004) betont zudem die Bedeutung relevanter Angebote im Internet für die jeweilige Gruppe als Voraussetzung für Zugang und Nutzung.

Iske et al. (2004) konstatieren einen voice divide bzw. voice inequality, um Fragen nach Ungleichheit in der aktiven Beteiligung im Internet, Interessenartikulation und der versuchten Einflussnahme der Nutzer(innen) untersuchen zu können. 
Hieraus ergibt sich ein komplexes, multidimensionales Bedingungsgefüge für die Nutzung des Internets, anhand dessen der Zusammenhang zwischen Internetnutzung und sozialer Ungleichheit weitreichend erfasst wird. Die empirische Umsetzung allerdings, die auch die Wechselwirkungen zwischen unterschiedlichen Differenzkategorien berücksichtigt, steht noch aus.

\subsection{Materielle und diskursive Konstruktionsprozesse: Diskriminierung durch stereotype Einschreibungen}

Zur Frage der sozialen Konstruktion und diskursiven Herstellungsweise des Internets kann insbesondere die Techniksoziologie auf die lange Tradition der sozialkonstruktivistischen Forschung und der Science and Technology Studies zurückblicken. Diese Forschungsrichtungen gehen davon aus, dass Technikentwicklung nicht einer Eigenlogik oder einem „one best way“ folgt, sondern dass bei der Konstruktion von Technik eine hohe „interpretative Flexibilität“ besteht und Technikentwicklung als Aushandlungsprozess zwischen den relevanten sozialen Akteur(inn)en zu betrachten ist (Pinch/ Bijker 1987). In diesen Aushandlungsprozess gehen die Vorstellungen der Beteiligten über zukünftige Nutzungsweisen ein und vergegenständlichen sich schließlich in einem Artefakt. Dabei können auch soziale Ungleichheit und Diskriminierungsstrukturen in Technik eingeschrieben werden. Winner (1985) verdeutlicht beispielsweise rassistische Ausschlüsse am Beispiel der New Yorker Brücke nach Long Island, die für PKWs befahrbar, für Busse allerdings zu niedrig gebaut war, so dass insbesondere die arme, überwiegend afro-amerikanische Bevölkerung New Yorks nicht in das Naherholungsgebiet gelangen konnte. Und von feministischen Techniksoziolog(inn)en wurde anhand einer Reihe von Beispielen herausgearbeitet, inwiefern in technische Artefakte geschlechterstereotype Handlungsvorschläge eingeschrieben sind (u. a. van Oost 2003).

In der Internetforschung sind sozialkonstruktivistische Perspektiven überraschend wenig prominent (Ausnahmen u. a. Kubicek/Dutton/Williams 1997, Thomas/Wyatt 1999, Paulitz 2005). Dabei enthält auch das Internet eingeschriebene Vorstellungen und Bilder und ist Gegenstand diverser Aushandlungsprozesse (z. B. Carstensen 2009). Auch zeigt beispielsweise die Debatte um barrierefreies Internet, dass u. a. Menschen mit Sehbeeinträchtigungen zunächst nicht als Nutzer(innen) bedacht wurden. Wötzel-Herber (2008) untersucht die Anmeldeformulare und Pflichtfelder in den Persönlichkeitsprofilen sozialer Netzwerke auf die (impliziten) Vorstellungen der Programmierer(innen). Und Rommes/van Oost/Oudshoorn (1999) analysieren das Design der Digital City of Amsterdam. Aber nicht nur technisches Design kann ausgrenzend sein, auch die Inhalte des Internets können soziale Ungleichheit (re-)produzieren. So zeigen frühe Untersuchungen beispielsweise, dass die Inhalte zunächst vor allem an männlichen Interessen orientiert waren: Auto, Computer, Sport und Pornografie machten einen Großteil der Internet-Angebote aus (Winker 2002).

Bemerkenswert sind zudem auch die zahlreichen Diskurse, die sich um das Internet von Beginn an rankten und neben mächtigen Symbolen, Mythen und Metaphern Vorstellungen von „richtigen“ Nutzungsweisen, Eigenschaften und Auswirkungen des Internets etablierten (u. a. Neverla 1998). In diesem Zusammenhang ist beispielsweise interessant, dass das Internet in manchen Kontexten als Männerdomäne entworfen wurde, verbunden mit einem Bild von Frauen als technikfern, ängstlich und zögerlich im Umgang mit Technik (Carstensen 2008). Hacker (2007) zeigt an Texten und Bildern aus entwicklungspolitischen Diskursen zum digital divide Prozesse sozialer Minorisierung aus feministischer und postkolonialer Perspektive auf. Auch auf symbolischer Ebene finden somit Ausgrenzungen und die Reproduktion von sozialer Ungleichheit statt. 


\section{4 „Cyberspace“ als neue Welt: Nene Freibeiten und alte Zwänge bei Identitätsexperimenten}

Ein weiteres Forschungsfeld betrifft schließlich Fragen nach Qualität und Beschaffenheit der so genannten „virtuellen Realität“, „virtueller Gemeinschaften“ bzw. des „Cyberspace" (u. a. Rheingold 1994, Becker/Paetau 1997, Turkle 1998, Stegbauer 2001, Thiedeke 2004). Für die Frage nach sozialer Ungleichheit ist in diesem Forschungsstrang interessant, inwiefern diese im „Cyberspace“ reproduziert wird (u. a. Bühl 1998, Thiedeke 2004) und wie viel Freiheit Subjekte haben, sich bzw. ihre Identitäten jenseits von Differenzkategorien neu zu entwerfen. Die Möglichkeiten anonymer und körperloser Kommunikation im Netz führten schon früh zu Debatten um Identitätsexperimente, Geschlechtertausch, fragmentierte Identitäten und neue Erfahrungsräume. Turkle (1998) beispielsweise sah zunächst diverse neuartige Möglichkeiten, die eigene Selbstdarstellung und Identitätskonstruktion beliebig zu gestalten. Das Internet stelle einen neuen Wahrnehmungsraum dar, in dem Identitäten jenseits der sichtbaren Körpermerkmale wie Geschlecht, Hautfarbe oder Alter frei erfunden werden können, wodurch diese Ungleichheit konstituierenden Faktoren an Relevanz auch in der "realen“ Welt verlieren könnten. Schachtner (2009) sieht im „Cyberspace“ sowohl Verfestigungs- als auch Wandlungstendenzen tradierter Geschlechterverhältnisse. Wötzel-Herber (2008) sowie Manago u. a. (2008) zeigen, inwiefern „reale“ Identitätskategorien in Social Networking Sites bei der Gestaltung der Persönlichkeitsprofile von zentraler Bedeutung sind. Insbesondere Geschlecht wird hier in sehr stereotypen Repräsentationen reproduziert. Andere Studien illustrieren, wie Hierarchien und Machtstrukturen an spezifischen Orten des Internets neu errichtet werden. So untersucht Stegbauer (2009) beispielsweise die Herausbildung von Kooperationsstrukturen und Machtpositionen innerhalb der Online-Enzyklopädie Wikipedia.

Insgesamt geht es somit zentral um die Frage, inwiefern sich der „Cyberspace“ hinsichtlich der Reproduktion von sozialer Ungleichheit von der „realen Welt“ unterscheidet, wobei ein Schwerpunkt auf Fragen nach Identitätskonstruktionen liegt.

\subsection{Zwischenfazit}

Wenngleich in dieser Zusammenschau deutlich wird, dass Forschungsfragen nach sozialer Ungleichheit und Diskriminierung in der Internetforschung einen prominenten Platz einnehmen, zeigt sich gleichzeitig auch, dass die einzelnen Forschungsstränge auf unterschiedlichen Ebenen argumentieren. Behandeln Forschungen zum digitale divide in erster Linie (sozial-)strukturelle Themen (z. B. Stadt-Land-Unterschiede im Internetzugang), fragen die konstruktivistisch angelegten Studien zur Herstellung des Internets vor allem nach Diskriminierungen durch Stereotype, Bilder und Normen (z. B. geschlechterstereotype Avatar-Figuren). Demgegenüber stehen in Untersuchungen, die sich der Beschaffenheit des „Cyberspace“ widmen, Fragen nach Identitätsentwürfen im Netz im Vordergrund. Wie diese Ebenen zusammenhängen, wird dabei selten geklärt. Am ehesten gelingt es noch der Nutzungsforschung, das komplexe Gefüge aus Faktoren wie Medienkompetenz, Motivation, Interesse auf der individuellen Ebene und Faktoren wie Geld, Zeit, technischer Infrastruktur und Unterstützungsstrukturen auf der strukturellen Ebene zusammenzudenken. Allerdings werden hierbei meist diskursive und normative Einflüsse vernachlässigt, obwohl in Interviews schnell deutlich wird, dass beispielsweise Diskurse zu Datenschutz, dem potenziellen Arbeitgeber, der Informationen über seine Bewerber(innen) im Web sucht, oder zu Suchtgefahren des Internets das Nutzungsverhalten maßgeblich mitprägen. Die Zusammenhänge zwischen struktu- 
reller, repräsentativer und identitärer Ebene werden in der Internetforschung kaum untersucht. Ebenso wenig wird in der Regel die Verwobenheit verschiedener Differenzkategorien herausgearbeitet. Zwar liegen auf struktureller Ebene Analysen vor, die verdeutlichen, dass es hinsichtlich des Internetzugangs Wechselwirkungen zwischen verschiedenen Kategorien gibt, doch wird dieser Zusammenhang nicht qualitativ beschrieben. Wie aber ist eine Analyse entlang unterschiedlichster Differenzkategorien und auf den drei genannten Ebenen theoretisch und empirisch denkbar?

\section{Soziale Ungleichheit erforschen: Intersektionale Mehrebenenanalyse}

Eine Antwort auf die Frage, wie sich soziale Ungleichheit auch im Internet analysieren lässt, sehen wir im Konzept der Intersektionalität. Der aus den USA kommende Ansatz versucht, mit Kategorien wie race, class, gender und sexuality verschiedene Dimensionen sozialer Ungleichheit und kultureller Verschiedenheit in integrativer Perspektive zu behandeln. Ein Ausgangspunkt ist die Erkenntnis, dass sich soziale Differenzierungen entlang unterschiedlicher Kategorien überlagern und nur unter Berücksichtigung ihres spezifischen Zusammenwirkens angemessen begriffen werden können. Intersektionalität wird seit den 1990er Jahren insbesondere in der Geschlechterforschung breit diskutiert. Dort ist mit der Kritik an den blinden Flecken durch postkoloniale, behinderte und queere Feministinnen deutlich geworden, dass neben Geschlecht auch andere Differenzkategorien bedeutsam sind und in bestimmten Kontexten sogar deutlich relevanter sein können. Darüber hinaus wurde klar, dass sich Ungleichheit generierende Kategorien auch nicht im Sinne einer „Mehrfachunterdrückungsthese“ einfach addieren lassen. Denn sie können sich wechselseitig verstärken oder auch abschwächen. So verstärkt beispielsweise das Alter die Wahrscheinlichkeit, dass Menschen keinen Zugang zum Internet finden, was insbesondere für Frauen zutrifft. Gleichzeitig kann dieser Ausschluss mit hoher Bildung ausgeglichen oder durch fehlende Sprachkompetenz verschärft werden. Wie sich diese Überlagerungen unterschiedlicher Diskriminierungsfaktoren darstellen, muss im jeweils konkreten Kontext untersucht werden.

Derzeit ist das Konzept der Intersektionalität auf dem besten Weg, zu einem neuen Paradigma in den Gender und Queer Studies zu avancieren (Knapp 2005, vgl. auch Bührmann 2009), da sich darüber vielfältige Differenzierungskategorien wie beispielsweise Gesundheit, Alter, Religionszugehörigkeit, Hautfarbe, Ethnizität und Nationalität integrieren lassen. Das Konzept der Intersektionalität ist darüber hinaus offen für konstruktive Weiterentwicklungen und Anwendungen aus verschiedenartigen Perspektiven und verfügt damit über ein Strömungen übergreifendes Potenzial (Davis 2008).

Auch wenn mittlerweile anerkannt ist, dass Kategorien wie Sexualität, Klasse, Alter, Bildung, Ethnie mit Geschlecht verwoben sind, wird oftmals nicht expliziert, wie diese Wechselwirkungen theoretisch und empirisch zu fassen sind und auf welchen Ebenen sich soziale Ungleichheit intersektional analysieren lassen. Mit der intersektionalen Mehrebenenanalyse machen Gabriele Winker und Nina Degele (2009) einen Vorschlag, wie zum einen die Wechselwirkungen zwischen Ungleichheit generierenden Kategorien und zum anderen gleichzeitig auch die Wechselwirkungen zwischen drei Ebenen - nämlich von Sozialstrukturen, symbolischen Repräsentationen und Identitätskonstruktionen - untersucht werden können. Die wichtigsten Bausteine dieses Ansatzes werden im Folgenden kurz dargestellt. 


\subsection{Soziale Praxen}

Den zentralen Ausgangspunkt der intersektionalen Mehrebenenanalyse stellen, mit Bezug auf die Theorie der Praxis von Bourdieu, die empirisch erfassbaren sozialen Praxen dar. Winker/Degele (2009: 63ff.) analysieren mit ihrem praxeologischen Ansatz das auf Körper, Sprache und Wissen basierende Handeln von Menschen. So werden konkrete Praxen Einzelner als Ungleichheit bedingende und bedingte sichtbar.

Praxistheoretische Konzepte sind hinsichtlich der Technik- und Medienforschung deshalb interessant und anschlussfähig, da sie die Materialität sozialer Praxen in ihrer Abhängigkeit von Körpern und Artefakten berücksichtigen. Praxen werden hierbei als Know-how-abhängige Routinen aufgefasst, die sowohl von Körpern als auch von Artefakten in routinisierten Beziehungen beeinflusst und getragen werden (Reckwitz 2003: 289; Hörning/Reuter 2004). Damit wird zum einen der herausgehobene Stellenwert der Artefakte für soziale Praxen, zum anderen ihr konstruierter Charakter betont. Artefakte ermöglichen und begrenzen erst bestimmte Verhaltensweisen und sind dabei weder nur erleichternde Hilfsmittel noch totalisierende, determinierende Fakten. Sie werden in Abhängigkeit von den Wissensbeständen, Deutungsmustern und Lebensstilen der Subjekte sowie von den dominanten gesellschaftlichen Diskursen und Normen kulturalisiert (Hörning 2001). Praxen erfordern immer eine Ausstattung, eine „materielle Konfiguration", die ihnen zugrunde liegt und sie rahmt; sie inkorporieren notwendigerweise immer Objekte (auch Wehling 2006).

\subsection{Strukturen, Repräsentationen und Identitäten}

Im Anschluss an wissenschaftstheoretische Überlegungen von Sandra Harding (1991: 53ff) schlagen Degele/Winker (2009) eine Mehrebenenanalyse auf der Grundlage folgender Unterscheidung vor: Sie berücksichtigen sowohl soziale Strukturen inklusive Organisationen und Institutionen (Makro- und Mesoebene) sowie Prozesse der Identitätsbildung (Mikroebene) als auch kulturelle Symbole (Repräsentationsebene). Diese Unterscheidung von Identitäts-, Repräsentations- und Strukturebene ist am ehesten aus der Geschlechterforschung heraus zu verstehen, da sie ein Versuch ist, die drei zentralen Ansätze der Geschlechterforschung der letzten Jahrzehnte zu bündeln und zu verbinden. So greifen Winker/Degele (2009) erstens auf die strukturorientierte Feminismusdebatte der 1970er und 1980er Jahre zurück, die in der Debatte um Herrschaftsverhältnisse das Verhältnis von Kapitalismus und Patriarchat im Blick hat. Zweitens berücksichtigen sie die identitätsbezogene ethnomethodologisch orientierte Debatte um doing gender oder doing difference der 1980er Jahre. Im Gegensatz zur strukturorientierten Gesellschaftskritik geht es dabei sehr viel mehr um die konkreten Prozesse, wie Identitäten gemacht und dabei Differenzkategorien reproduziert werden. Und drittens beziehen sie sich auf die poststrukturalistische Debatte um das performative Hervorbringen und Verfestigen von Normen und Werten rund um das Werk von Judith Butler (1991) seit den 1990er Jahren. Damit erheben Winker/Degele (2009) den Anspruch, statt aus einer, aus drei Perspektiven auf gesellschaftliche Konstruktionsprozesse sozialer Ungleichheit zu blicken und damit diese umfassender analysieren zu können.

Dass diese Verbindung auch für die Internetforschung ein analytischer Gewinn sein kann, hat sich bereits im vorangegangenen Abschnitt gezeigt (vgl. 1.5): Die Analysen bewegen sich derzeit je nach theoretischer Ausrichtung und Interesse auf jeweils nur einer, maximal zwei der genannten Ebenen, die darüber hinaus meist unverbunden bleiben. 


\subsection{Auswabl der Kategorien}

Ein zentraler Punkt in der Intersektionalitätsdebatte ist die Frage nach den zu berücksichtigenden Kategorien. Während Klasse, race/Ethnie/Herkunft/Nationalität und Geschlecht als Dreiklang relativ unbestritten sind, fordern unterschiedliche Autor(inn)en die Erweiterung um jeweils verschiedenste Kategorien. Queere Forschung beispielsweise betont die Bedeutung der Kategorie desire (Butler 1991) bzw. Sexualität (u. a. Hark 1993), aus den disability studies kommt die Forderung nach Berücksichtigung von Krankheit bzw. Behinderung (u. a. Oliver 1996, Albrecht 2006, Jacob/Köbsell/Wollrad 2010). Lutz/Wenning (2001) benennen 13 bipolare hierarchische Differenzlinien - Geschlecht, Sexualität, „Rasse“/Hautfarbe, Ethnizität, Nation/Staat, Klasse, Kultur, Gesundheit, Alter, Sesshaftigkeit/Herkunft, Besitz, Nord-Süd/Ost-West und gesellschaftlicher Entwicklungsstand - als relevant für die Intersektionalitätsdebatte.

Winker/Degele (2009: 81ff.) schlagen hingegen für die Identitäts- und Repräsentationsebene ein induktiv offenes Vorgehen vor. Dies dient dazu, nicht vorschnell bekannte Kategorien zu reifizieren und beispielsweise Frauen wieder nur auf frauenspezifische Eigenschaften festzulegen. Sie gehen von einer nach oben offenen Anzahl von Kategorien aus, um verschiedenartige Identitätskonstruktionen und unterschiedliche Normen, Werte und Ideologien und auch Verweise auf soziale Strukturen in ihrer Vielfalt zu berücksichtigen und auch offen für neue, unerwartete Ungleichheitskategorien zu sein. Genauer gesagt setzen sie an konkreten Phänomenen an und analysieren sie u. a. in Hinblick auf dabei wirksame Ungleichheitsdimensionen. Es geht darum, die Ungleichheitserfahrungen aus Sicht der Subjekte zu rekonstruieren, um die Frage, wie Subjekte Ausgrenzung und Diskriminierung erleben, wahrnehmen und sich auch dagegen zur Wehr setzen.

Auf der Strukturebene legen Winker/Degele (2009: 37) demgegenüber vier zentrale Herrschaftsverhältnisse fest: Klassismen, Heteronormativismen, Rassismen und Bodyismen. Die Kategorien Klasse, Geschlecht (inkl. Sexualität) und Rasse/Ethnie ergänzen sie damit um die Kategorie Körper, um zu betonen, dass soziale Ungleichheit auch über körperliche Leistungsfähigkeit (Attraktivität, Gesundheit/Krankheit, Alter etc.) konstituiert wird. Die strukturellen Herrschaftsverhältnisse sollen nicht statisch auf die Auswertung angewendet werden, sondern es ermöglichen, die von Interviewpersonen genannten sozialen Strukturen mit den Erkenntnissen zu Klassen-, Geschlechter-, Rassen- und Körperverhältnissen in Beziehung zu setzen.

\section{4 Übersetzung in ein methodisches Vorgehen}

Um diesen Theorieansatz in der empirischen Forschung anwenden zu können, schlagen Winker/Degele (2009) acht Schritte vor (vgl. Abschnitt 3), anhand derer rekonstruiert wird, wie sich die Akteure und Akteurinnen selbst begreifen, wo sie Hindernisse für die Realisierung ihrer Lebensinteressen sehen, wo sie Unterdrückung und Diskriminierungen erfahren und wo sie auch Widerstandspotenziale erkennen.

So wenig intersektionale Fragestellungen bisher in der Internetforschung bearbeitet wurden, so wenig hat sich umgekehrt auch die Forschung zu Intersektionalität um technisch-mediale Tatbestände gekümmert. Fragen nach Diskriminierungen und Ausschlüssen wurden bisher nicht in den Kontext von Internet und anderen Artefakten gestellt, obwohl ihre Gesellschaft und damit Ungleichheit konstituierenden Effekte unbestritten sind.

Um dieses beidseitige Desiderat zu bearbeiten, stellen wir im folgenden Abschnitt unseren Vorschlag für ein intersektionales Forschungsdesign für die sozialwissenschaft- 
liche Internetforschung vor. Entlang der acht Schritte illustrieren wir an einem Fallbeispiel, mithilfe welcher Methoden die Verwobenheiten und Wechselwirkungen zwischen verschiedenen Ungleichheitskategorien, den unterschiedlichen Ebenen Identität, Repräsentation und Struktur sowie den vier Forschungssträngen der Internetforschung (Zugang, Nutzung, Konstruktion und „Cyberspace“) erforscht werden können.

\section{Intersektionale Mehrebenenanalyse in der Internetforschung: Vorschläge für ein Forschungsdesign}

Winker/Degele (2009: 80) schlagen für das methodische Vorgehen folgende acht Schritte vor:

- Block I: Auswertung einzelner Fälle (anhand von Interviewmaterial, Beobachtungen, Webanalysen etc.):

1. Identitätskonstruktionen beschreiben,

2. Symbolische Repräsentationen identifizieren,

3. Bezüge zu Sozialstrukturen finden,

4. Wechselwirkungen zentraler Kategorien auf drei Ebenen benennen $\rightarrow$ Bildung von Subjektkonstruktionen (auch Winker/Degele 2011).

- Block II: Analyse aller Fälle einer Untersuchung:

5. Identitätskonstruktionen vergleichen und clustern,

6. Strukturdaten ergänzen und Herrschaftsverhältnisse analysieren,

7. Analyse von benannten Repräsentationen vertiefen,

8. Wechselwirkungen in der Gesamtschau herausarbeiten.

Um soziale Ungleichheit im Internet angemessen zu untersuchen, ist es notwendig, mit unterschiedlichen Erhebungsmethoden zu arbeiten. Im Zentrum stehen dabei qualitative Interviews, die mit Webanalysen, teilnehmenden Beobachtungen von Internetpraktiken, Diskursanalysen und Strukturdatenanalysen kombiniert werden können. Im Folgenden skizzieren wir einen Vorschlag für ein Forschungsdesign und illustrieren ihn, wo möglich, mit Beispielen aus einem laufenden Forschungsprojekt ${ }^{1}$, in dem wir intersektional arbeiten. Entlang der acht Schritte werden jeweils die unterschiedlich einzusetzenden Methoden vorgestellt, deren Reichweite diskutiert und an dem Fallbeispiel verdeutlicht.

\section{Block I: Schritt 1-4}

Ausgehend vom empirischen Handeln und Sprechen von Subjekten schlagen Winker/ Degele (2009) vor, in einem ersten Schritt die Identitäten der untersuchten Personen herauszuarbeiten, die jene über Differenzierungskategorien herstellen. Daran anschließend arbeiten sie in einem zweiten Schritt heraus, auf welche Normen, Leitbilder und Deutungsmuster sich die untersuchten Personen beziehen, und in einem dritten Schritt verdeutlichen sie, auf welche sozialen Strukturen die Untersuchungspersonen verweisen. Das heißt, in diesen ersten drei Schritten wird die Selbstpositionierung von Subjekten rekonstruiert. Im vierten Schritt werden die Wechselwirkungen zwischen diesen drei Ebenen herausgearbeitet. Während diese Schritte bei der Analyse (z. B. bei der Codierung eines Interviews) trennbar sind, werden sie in der folgenden Darstellung immer gleich auch mit ihren Wechselwirkungen untereinander dargestellt, d. h. die Schritte 1-4 werden nicht völlig voneinander getrennt aufgeführt.

1 Teilprojekt „Webbasierte Erwerbsarbeit“ im Verbundprojekt „Subjektkonstruktionen und digitale Kultur“, gefördert von der Volkswagen-Stiftung, http://skudi.net. 
Auch im Feld Internetforschung eignen sich zunächst qualitative Interviews als Grundlage für die intersektionale Mehrebenenanalyse. Mit Auswertung der Interviews lässt sich erkennen, wie die Interviewten sich selbst (eventuell auch im „Cyberspace“) sehen (neugierig auf Technik, kommunikativ, ausgeschlossen, pragmatisch, hinterherhinkend, Pionierin, überfordert, vernetzt, jung, Grenzgänger etc.) und auf welche sozialen Strukturen (Arbeitsmarkt, technische Möglichkeiten, Gesetze, unzugängliche Bereiche im Netz etc.) und symbolischen Repräsentationen (Erreichbarkeitszwänge, Datenschutzdiskurse, Nutzungsnormen) sie verweisen. Damit kann anhand des Interviewmaterials auch herausgearbeitet werden, welche Bedeutungen das Internet im Alltag der Interviewten zugewiesen bekommt. Qualitative Interviews bieten damit zunächst die Grundlage für die Berücksichtigung aller drei Ebenen bei der Auswertung; gleichzeitig liefern sie Daten zu allen vier Bereichen der Internetforschung, vor allem aber zum Bereich der Nutzung (z. B. zu Motivationen, Kompetenzen, Interessen). Allerdings können an dem Interviewmaterial immer nur die Sichtweisen und Sinnhorizonte der Interviewten herausgearbeitet werden.

Unser Fallbeispiel - Paula - ist eine von 30 Interviewten aus unserem Forschungsprojekt zu webbasierter Erwerbsarbeit. Wir haben sie in einem Leitfaden-gestützten Interview zu ihren Erfahrungen mit dem Internet, ihrer Erwerbstätigkeit, ihrem Alltag und ihren Zukunftsplänen befragt. Sie ist 26 Jahre alt und hat mit mehreren Freundinnen und Freunden eine crossmedial arbeitende Internetagentur aufgebaut. Sie hat ein sozial- und geisteswissenschaftliches Studium mit der Note „sehr gut“ abgeschlossen und beschreibt sich selbst als jung, ehrgeizig und zielstrebig. Ein zentrales Thema im Interview mit Paula ist ihr Verhältnis zu den permanenten technischen Neuerungen und Angeboten im Netz.

Diesen technischen Wandel beschreibt sie auf der Strukturebene als schnell, unberechenbar und unkontrollierbar, wobei sie damit insbesondere die sozialen Netzwerke wie MySpace, Facebook und Twitter meint: "Also es ist halt am Anfang noch so 'n bisschen ungewohnt alles und man kommt damit gar nicht so richtig klar, und dann hat man das drauf, dann kommt wieder was Neues". Sie beschreibt es als anstrengend, dass man nie wisse, was als nächstes komme und wie lange sich der Hype halte: „also, irgendwie hört man, da ist was mit Twitter, dann macht man's und plötzlich, ehe man sich versiebt, macht es jeder, genauso schnell kann's ja wieder vorbei sein".

Auf der Repräsentationsebene nimmt sie sehr deutlich einen Zwang zum „Mitmachen“ und „Mithalten“ wahr sowie die Notwendigkeit, sich im Netz öffentlich zu präsentieren, "man ist einfach da der Präsenz wegen, weil man's ja irgendwie sein muss, weil wir sind ' $n$ Internetunternebmen, wir können nicht nicht auf Facebook sein und nicht nicht auf Twitter und, am besten entwickeln wir noch ' $n$ App". Es scheint somit selbstverständlich zu sein und erwartet zu werden, dass jede technische Neuerung auch genutzt werden müsse.

Sich selbst positioniert sie als kritisch diesem Wandel gegenüber, sie stellt den Nutzen und die Sinnhaftigkeit in Frage, ständig neue, zumal ähnliche Angebote nutzen zu müssen: „wobei man sich manchmal auch fragt, muss das denn eigentlich sein, also auf Facebook kann man's genauso machen oder auf MySpace, warum gibt's dann noch Twitter". Diese Zweifel am Sinn und Zweck werden von der Norm, jedes neue Tool nutzen zu müssen, konterkariert: „also, das sind so Sachen, wo man manchmal sich tatsächlich über die Sinnhaftigkeit, also wo ich mir nicht so ganz über den klaren Nutzen bewusst bin, es aber einfach mache, weil ich denke, man muss es ja auch". Sie beschreibt, dass man sich dem Druck nicht entziehen kann, beschreibt dies als anstrengend, sie fühlt sich getrieben und gestresst. Zwar nimmt sie sich immer wieder vor, mehr als das, was sie jetzt nutzt, nicht mehr zu nutzen, aber dies durchzuhalten, gelingt nicht: „wo ich auch am 
Anfang dachte, mehr als heute online xy mach'ich auch nicht, mach'ich dann aber doch, also man entwickelt dann, sucht sich doch so seine Nischen". Gleichzeitig beschreibt sie ihre Strategien, sich immer wieder in neue Angebote „reinzufinden“ und passende Nutzungsweisen zu erproben und zu entwickeln: „da muss man sich dann erstmal reinfinden, wie ist das Freundschaftsanfragen, obh thanks for the add und so, und dann fänt man da so an, also ganz ' $n$ bisschen, so dann poste ich mal da was".

Dieses kurze Beispiel verdeutlicht bereits, wie die drei Ebenen Identität, Repräsentation und Struktur schon in den einzelnen Textstellen miteinander verwoben sind und sich nicht nur einer Ebene zuordnen lassen. Die wachsende Zahl an sozialen Netzwerken ist nur deshalb - trotz fehlender Zweckmäßigkeit - für die Interviewte von Bedeutung und restriktiv, weil sie sich mit Diskursen und Nutzungsnormen verschränken, die es als Muss definieren, auf allen Netzwerken präsent zu sein. Zudem finden sich Hinweise auf die Verwobenheit der verschiedenen Forschungsstränge der Internetforschung: Es wird deutlich, wie mit der Herausbildung von Nutzungsweisen (sich immer wieder in Neues „reinfinden“, ausprobieren, bis es normal wird) auf materielle und diskursive Konstruktionsprozesse (das ständig wachsende Angebot an neuen Tools sowie der dazu gehörige Diskurs, diese nutzen zu müssen) reagiert wird. Insgesamt fällt gleichzeitig nicht nur an dieser Textstelle - auf, dass die „klassischen“ Differenzkategorien bzw. Herrschaftsverhältnisse nur sehr selten explizit von den Subjekten selbst thematisiert werden.

Für eine empirische Internetforschung sind neben Interviews Erbebungen von webbasiertem Material unerlässlich. Im Rahmen der Schritte 1 bis 3 sind hierfür, wenn vorhanden und zugänglich, die Selbstdarstellungspraxen auf eigenen Webseiten, Persönlichkeitsprofile in Social Network Sites, Nachrichten auf Weblogs oder Microblogs von Interesse. Über dieses Material können ebenfalls Identitätskonstruktionen der Interviewten sowie deren Sicht auf Sozialstrukturen, Normen und Diskurse ermittelt werden.

Am Beispiel Paula zeigt sich hier eine professionelle Selbstdarstellung im Netz, eine eigene Homepage mit Vita und Arbeitsproben. Über Informationen, Meldungen, Nachrichten oder Tweets auf sämtlichen sozialen Netzwerkseiten und Blog-Seiten wird die regelmäßige Vermarktung des eigenen Unternehmens deutlich. Private Informationen oder Nachrichten findet man nicht. Über die Präsentation als hochqualifiziert und die Zurschaustellung des eigenen beruflichen Lebenslaufs wird somit zumindest die Ungleichheitskategorie Bildung explizit betont. Von der Identitätsdarstellung als gut ausgebildet kann abgeleitet werden, dass in gewisser Weise die Norm eines qualifizierten Lebenslaufes sowie die Anforderung der Selbst-Vermarktung anerkannt und bedient wird, worin indirekt zudem strukturelle Bedingungen wie ein prekärer Arbeitsmarkt sichtbar werden.

Das heißt, auch hier können anhand der Selbstdarstellungen Aussagen zu den drei Ebenen Identität, Repräsentation und Struktur sowie die Nutzungsweisen der Subjekte herausgearbeitet werden. Zudem befinden sich Webanalysen von selbst produziertem Material im Netz an der Schnittstelle der zwei Internetforschungsstränge Konstruktion und Nutzung. Dabei wird zum einen deutlich, dass diese beiden Bereiche zunehmend typisch für das „Web 2.0“ - verwischen, und zum anderen kann herausgearbeitet werden, wie materialisierte Artefakte im Netz die individuellen Nutzungsweisen beschränken, z. B. über Formulare und Pflichtfelder, bzw. Handlungsspielräume eröffnen.

Interviews und auch Webanalysen von selbst produziertem Material geben allerdings vor allem Aufschluss über Bedeutungskonstruktionen und Sinnhorizonte aus Sicht der Subjekte und nur begrenzt über die konkreten Praxen, da Handeln und das Sprechen über das Handeln oftmals auseinanderfallen. „Man braucht, anders gesagt, eine Kon- 
trastfolie, etwas, mit dem sich das Gesagte vergleichend in Beziehung setzen lässt, damit erkennbar wird, was im Reden verdeckt bleibt“" (Wetterer 2003: 292). Dies gilt insbesondere für Forschungen, in denen technisch-mediale Gegenstände von Bedeutung sind. Es hat sich gezeigt, dass insbesondere im Umgang mit neuen Technologien oftmals die konkrete Sprache fehlt, also Begrifflichkeiten und Formulierungen fehlen, um alltägliche Routinen und Tätigkeiten sowie ungeübte Handlungen zu beschreiben (Carstensen/ Winker 2005b: 92). Daher arbeiten wir ergänzend mit der Aufzeichnung von Internetpraktiken. Hierfür werden die Interviewpersonen nach dem Interview gebeten, ihre alltäglichen Routinen am Computer durchzuführen sowie von uns vorgegebene Such-, Recherche- oder Programmieraufgaben zu bearbeiten, die mithilfe einer Software aufgezeichnet werden. Darüber können verkörperte und routinisierte Praxen im Umgang mit dem Internet unmittelbar erhoben werden. Die untersuchten Personen kommentieren dabei ihr Handeln mit dem Internet laut; diese Kommentare werden wie die Mimik (und begrenzt auch die Gestik) über Kamera und Mikrofon aufgezeichnet.

Mit den Daten, die durch diese Methode erhoben werden, wird zum einen die Subjekt-Technik-Interaktion mit ihren förderlichen, irritierenden, hinderlichen Elementen und Wechselwirkungen analysierbar; d. h. die Frage nach der Vermittlung zwischen Interfaces und Nutzungsweisen kann darüber beantwortet werden. Hinsichtlich der Ebenen kann somit vor allem das (verwobene) Verhältnis von Identität und (technischer) Struktur untersucht werden. Aussagen zur Repräsentationsebene sind über diese beobachtende Methode hingegen weniger zu gewinnen. Zudem ermöglicht dieses Vorgehen Aussagen über die Praxen der Nutzung (und gegebenenfalls des Konstruierens) des Internets, inklusive der die Nutzung bestimmenden Faktoren wie Medienkompetenz und Interessen. Auch verdeutlicht es den Umgang der Subjekte mit den in die Artefakte eingeschriebenen Herrschaftsverhältnissen. Theoretisch können in diesem Erhebungsschritt auch Einsichten in das Agieren im „Cyberspace“ entstehen, es kommt allerdings selten vor, dass die Interviewten dies in der Aufzeichnungssituation demonstrieren. Die Stärke dieser Methode liegt damit im Beobachten konkreter Praxen mit dem Internet.

Am Beispiel Paula zeigt sich im konkreten Umgang mit dem Internet, dass ihr alltägliches Handeln darin besteht, die selbstproduzierten Audio-, Video- und Textdaten hochzuladen, mit diversen Seiten zu verlinken und die Links auf Weblogs und Sozialen Netzwerkseiten zu posten. „Copy and Paste“ von HTML-Code stellt die zentrale Tätigkeit dar; Paula jongliert dabei geschickt und routiniert zwischen einer Vielzahl von Seiten hin und her. Von strukturell-technischer Seite wird deutlich, wie beschränkt der Handlungsspielraum an dieser Stelle ist, da sehr exakte Eingaben per Hand erforderlich sind, die sich zudem offensichtlich nicht automatisieren lassen. Das Internet fordert hier einen konzentrierten Umgang auf gleichzeitig niedrigem Komplexitätsniveau, vorausgesetzt, die grundlegende Logik von HTML ist bekannt. Die Eingabeformulare der aufgerufenen Seiten bieten dabei keinen Spielraum für Abweichungen; diese „Kompromisslosigkeit" kann bei Nichtbeherrschung von HTML ausgrenzend und zu einem Faktor sozialer Ungleichheit werden. Bildung bzw. Technikkompetenz werden als Anforderungen und damit als Ungleichheitskategorien sichtbar.

Deutlich wird auch zum einen auf Strukturebene sowie hinsichtlich der Konstruktion des Internets, dass gegenwärtig verschiedene Netzwerkseiten nebeneinander existieren, die alle - und das ist dann bereits der Übergang zur Repräsentationsebene (und der diskursiven Konstruktion des Internets) - gleichermaßen bedient werden müssen, will man sein Unternehmen vermarkten. Dieser Punkt ist bereits im Interview zum Ausdruck gekommen. Deutlicher als im Interview wird an der Beobachtung zum anderen, wie geschickt und kompetent Paula mit den diversen Tools umgeht, und dass das Genervt- 
und Gestresstsein vom ständigen Wandel und der Vielzahl an zu nutzenden Internetangeboten offenbar für sie nicht verhindert, praktikable und routinierte Nutzungsweisen zu etablieren.

Es ist also möglich, mit verschiedenen Methoden umfassendes Material zu den ersten drei Schritten der intersektionalen Mehrebenenanalyse zu erheben. Wie bereits deutlich wurde, sind die Ebenen Identität, Repräsentation und Struktur eng miteinander verwoben. Daher arbeiten Winker/Degele (2009: 86f.) in Schritt 4 die Wechselwirkungen zwischen diesen drei Ebenen heraus und kommen damit zu zentralen Subjektkonstruktionen. Diese sind eine Interpretationsleistung der Forscher(innen), die sich über die Analyse der Wechselwirkungen zwischen Identitätskonstruktionen, symbolischen Repräsentationen und sozialen Strukturen, wie bis hierin dargestellt, ergeben. Die Identitätskonstruktionen sind damit ein Teil der Subjektkonstruktionen; zusätzlich gehen aber auch die Bezugnahmen auf die Repräsentations- und die Strukturebene in die Subjektkonstruktionen ein.

D. h. an dieser Stelle werden aus Wechselwirkungen zwischen den Identitätskonstruktionen sowie den Sichtweisen der Interviewten auf symbolische Repräsentationen und soziale Strukturen zusammenfassende Aussagen gebildet. Für das Fallbeispiel Paula sind dabei zum Beispiel folgende Wechselwirkungen relevant: die zwischen dem schnellen technologischen Wandel auf der Strukturebene und dem Diskurs, der zum „Mitmachen“ und zur Präsenz auffordert; die zwischen ihrer Identitätskonstruktion als den Nutzen immer neuer Tools kritisch in Frage stellend und Nutzungsaufforderungen, denen sie sich dennoch nicht entziehen kann; ihre professionellen Praktiken der Selbstdarstellung im Netz als hochqualifiziert und ihr kompetentes Jonglieren mit HTMLCodes als Antwort auf den von ihr sowohl auf der Repräsentations- als auch auf der Strukturebene wahrgenommenen Druck. Diese Wechselwirkungen können, wenn möglich, auf einen Satz oder eine Formulierung zugespitzt werden. Für Paula könnte eine solche Subjektkonstruktion beispielsweise lauten: „Die von technischen Entwicklungen und Nutzungsanforderungen bedrängte und gestresste, aber kompetent-strategische Unternehmerin.“

\section{Block II: Schritt 5}

Handelt es sich um eine größere empirische Forschung mit vielen untersuchten Personen und umfangreichem Interviewmaterial, schlagen Winker/Degele (2009) vor, diese Subjektkonstruktionen im Schritt 5 zu typisieren. Hierfür sind zunächst wie bei jeder Typenbildung die zentralen Vergleichsdimensionen festzulegen (auch Kelle/Kluge 1999). Für unser Sample zu webbasierter Erwerbsarbeit ist hierfür beispielsweise, wie auch am Fall Paula deutlich wird, der Umgang mit dem technologischen Wandel eine Möglichkeit, nach der die Fälle strukturiert werden können. Andere Dimensionen in diesem Forschungsfeld könnten Arbeitsweisen, der Umgang mit Öffentlichkeit oder die unterschiedlichen Grade an Belastungen sein. Auch eine Typisierung entlang selbst thematisierter Diskriminierungen oder Ungleichheiten ist denkbar. Legen wir beispielsweise den Umgang mit technologischem Wandel als Vergleichsdimension zugrunde, können die Fälle danach sortiert werden, welche Bewältigungsstrategien die Interviewten entwickeln. Paula wäre dann ein Beispiel für eine kompetente, aber gestresst-genervte Aneignung, die, wenn sie sich durch weitere Fälle sättigen lassen würde, zum Beispiel den Typ des angestrengten Umgangs mit konstituieren könnte. Weitere Typen, die sich in unserem Sample zeigen und im Kontrast zu diesem ersten Typ stehen, zeigen einen leidenschaftlich-genussvollen Umgang mit technischen Neuerungen, wieder andere betrachten den technologischen Wandel als persönliche Gestaltungsaufgabe, sei es aus 
technischen oder politischen Motiven. Schließlich finden sich auch abgegrenzte, pragmatische bis hin zu desinteressierten Haltungen gegenüber dem technologischen Wandel, die sich wenig von diskursiven Nutzungsaufforderungen beeindrucken lassen, sondern ein sehr funktionales Verhältnis zu neuen Tools haben und sich diese nur aneignen, wenn sie einen Nutzen erkennen können.

Dieser Schritt entspricht weitgehend einer klassischen Typenbildung. Um die Verwobenheiten von Ungleichheiten und Diskriminierungen herauszuarbeiten, werden die Ergebnisse im Folgenden mit zusätzlichen Daten bereichert.

\section{Schritt 6 und 7}

Gehen Winker/Degele in den ersten fünf Schritten induktiv vor, so konfrontieren sie in den nächsten beiden Schritten die bis dahin erarbeiteten Ergebnisse mit theoriegeleiteten Vorgaben. So werden in Schritt 6 die Verweise der verschiedenen Subjektivierungstypen auf soziale Strukturen in den Zusammenhang gebracht mit den deduktiv gesetzten vier Herrschaftsverhältnissen, nämlich mit Klassismen, Heteronormativismen, Rassismen und Bodyismen. Damit lassen sich die Ergebnisse fokussieren und zuspitzen. Entsprechend werden in Schritt 7 die genannten symbolischen Repräsentationen mit hegemonialen Werten, Normen und Deutungsmustern konfrontiert, wie sie aus anderen Untersuchungen bekannt sind.

Neben der Hinzunahme von Theorien und Forschungsständen empfehlen sich an dieser Stelle speziell für die Internetforschung Strukturdatenanalysen, Webanalysen sowie Diskursanalysen, um die Bedeutung der vier Herrschaftsverhältnisse zu analysieren.

Strukturdatenanalysen: Während Winker/Degele (2009) für die Analyse auf der Strukturebene vor allem Organisationen und Institutionen im Blick haben, bieten sich im Bereich Internetforschung weitere Strukturdaten zur Analyse an. Je nach Forschungsfrage sind hierbei beispielsweise folgende Daten von Interesse: Gesetze zu Datenschutz, allgemeine Geschäftsbedingungen von sozialen Netzwerken, Vertrags- und Preisstrukturen von Providern sowie die Netzversorgung in der Region, aber auch Zugangs- und Nutzungsdaten verschiedener Internet-Angebote nach sozio-demografischen Daten aufgeschlüsselt. Für das angeführte Beispiel ist beispielsweise interessant, die Entwicklung der Nutzungszahlen der thematisierten Angebote (Facebook, MySpace, Twitter) zu analysieren, um die von Paula bzw. dem „angestrengten Typ“ wahrgenommene Geschwindigkeit der Durchsetzung neuer Angebote zu prüfen. Diese so gewonnenen Daten können bereits Verwobenheiten zwischen verschiedenen Ungleichheitsdimensionen auf der Strukturebene verdeutlichen, beispielsweise von Geschlecht, Region und Alter hinsichtlich des Internetzugangs zu neuen Angeboten, ähnlich wie unter 1.1 und 1.2 bereits aufgezeigt wurde.

Webanalysen können Aufschluss über die Angebotsstruktur im Netz für eine bestimmte Zielgruppe (z. B. Erwerbslose, Alleinerziehende) geben. Dabei geht es nicht um reine Inhaltsanalysen, sondern auch um die Auswertung der technischen Möglichkeiten bzw. der in Artefakten materialisierten Klassismen, Bodyismen etc. (die z. B. deutlich werden an Interaktionsmöglichkeiten, Formularen, Kommentarfunktionen, Barrierefreiheit, Userfreundlichkeit, Sprachen, Zugangsbarrieren). D. h. es ist zu untersuchen, was bestimmte (viel genutzte) Websites ermöglichen, was sie beschränken, inwiefern sie diskriminierend sind und wie viel Raum sie für die Darstellung von Identitätskonstruktionen lassen. Auch können anhand von Kommentaren in Weblogs, von Gruppen in Social Networks oder anhand von Repräsentationen in Avataren (z. B. in Second Life) materialisierte Repräsentationen im Netz analysiert werden. Diese Analysen sind sowohl auf der strukturellen als auch auf der normativ-diskursiven Ebene angesiedelt und 
zeigen, inwiefern die in Formularen, virtuellen Umgebungen und Kommunikationsräumen materialisierten Herrschaftsverhältnisse auf Zugang und Nutzung wirken. Für das vorgestellte Sample sind hierbei beispielsweise Analysen der Interfaces von sozialen Netzwerkseiten relevant, die auf ihre Voraussetzungen, Komplexität, Möglichkeiten der Mitgestaltung etc. hin analysiert werden.

Um die in den Interviews identifizierten Repräsentationen mit den hegemonialen Diskursen abzugleichen, können zudem Diskursanalysen zu den angesprochenen Themen durchgeführt werden; in Bezug auf das Internet sind hierbei beispielsweise Datenschutz- oder Suchtgefahrdiskurse zu nennen, auf die in vielen Interviews verwiesen wird. Auch hierbei gilt das besondere Interesse der Frage, wie weitgehend diese Diskurse mit den vier Herrschaftsverhältnissen verwoben sind. In Bezug auf die in unserem Sample relevante Dimension des Umgangs mit technologischem Wandel ist besonders von Interesse, welche Nutzungsaufforderungen zu neuen Tools sich in Printmedien finden, aber auch in Foren, Weblogs und sozialen Netzwerken verhandelt werden, und wer dort wie adressiert wird.

\section{Schritt 8}

Über die Hinzuziehung externen Materials sollen damit explizit auch die vier Herrschaftsverhältnisse an die Ergebnisse angelegt werden. So können auch nicht thematisierte Ungleichheitskategorien auf ihre Relevanz befragt werden. Die Ergebnisse werden in Schritt 8 abschließend zusammen betrachtet. Hier geht es wieder um die Wechselwirkungen der drei Ebenen Identität, Repräsentation und Struktur. Die Einwirkungen aufeinander können von unterschiedlicher Form sein, sie können etwa abschwächende, verstärkende oder auch neutrale Wirkungen in Form von Persistenz, Modifikation oder auch fehlende Resonanz zeitigen (Winker/Degele 2009: 79ff.).

So ist beispielsweise bemerkenswert, dass die Kategorie Geschlecht in den Interviews so gut wie keine Rolle spielt, weder bei den weiblichen noch bei den männlichen Interviewten. $\mathrm{Ob}$ sie deshalb tatsächlich irrelevant ist oder ob hier Herrschaftsverhältnisse dethematisiert werden, könnte dann anhand von Forschungsergebnissen und Theorien zum Verhältnis von Gender und Technik sowie zur Situation von Frauen in der Internetbranche diskutiert werden.

Sehr deutlich werden hingegen klassistische Herrschaftsverhältnisse. Bereits an Paulas Äußerungen wurde deutlich, dass Bildung für sie eine zentrale Kategorie darstellt, die im Kontext des Internets und webbasierter Erwerbsarbeit privilegiert bzw. benachteiligt. Darüber hinaus zeigt sich insbesondere Sprache als wichtige Kompetenz im Internet. Sie ist Voraussetzung für Kommunikation, für eine überzeugende Selbstdarstellung auf Homepages und Netzwerken oder beim Bloggen und verweist sowohl auf klassistische als auch auf rassistische Herrschaftsverhältnisse, da je nach Kontext das Bildungsniveau und die Muttersprache bzw. die Herkunft über Sprach- und Ausdruckskompetenz ausgrenzend wirken können. Auch zeigt sich, wie wichtig Medienkompetenz geworden ist, zum einen in Form grundlegender HTML-Kenntnisse u. ä., zum anderen im Sinne der Kompetenz, sich permanent in neue Technologien einzuarbeiten und sich diese anzueignen. Sowohl Struktur- als auch Diskursanalysen stützen diesen Befund. „Medienkompetenz“ gilt als Schlüsselkompetenz und hat sich mit dem Internet zu einem normativen Konzept entwickelt, das darauf zielt, dass Subjekte handlungsfähig gegenüber Technik werden (Hurrelmann 2002; Paulitz 2005). Sich regelmäßig die neueste Technologie anzueignen, ist vorausgesetzte Norm geworden. Dabei zeigt sich auch, dass es dabei nicht mehr nur um die Frage nach Zugang oder Nicht-Zugang geht, sondern dass Ausschlüsse und digitale Gräben auch innerhalb der Internetnutzer(innen) entste- 
hen und zwar entlang der Frage der Nutzung bzw. Nicht-Nutzung aller „wichtigen“ Angebote.

Und schließlich zu Bodyismen: Paula selbst betont im Interview bereits ihr Jungsein. In Schritt 6 und 7 können Struktur- und Diskursdaten belegen, dass Alter eine der zentralen Kategorien ist, die im Internet für Ungleichheit sorgt. Die Aufzeichnungen der Internetpraktiken verdeutlichen zudem körperliche Kompetenzen wie Geschicklichkeit und Schnelligkeit im Umgang mit der Tastatur als Vorteile und zeigen zudem, wie inkorporiert und selbstverständlich der körperliche Umgang mit dem Internet sein kann. Weiterführend könnten hier zudem Analysen der Hardware (Tastengröße etc.) sein.

So lässt sich in diesem Schritt zeigen, welche Bedeutung die Kategorien Bildung, Sprache und Alter im Bereich webbasierter Erwerbsarbeit haben. Zudem wird das Wechselverhältnis von technischen Strukturen und den jeweils gültigen (Nutzungs-)Diskursen deutlich, die erst gemeinsam ihre Wirkmächtigkeit entfalten können. An dem Fallbeispiel dürfte zudem insbesondere die Verwobenheit der Internetforschungsbereiche Nutzung und Konstruktion deutlich geworden sein.

Insgesamt dominieren klassistische Herrschaftsverhältnisse die Situation von Paula, was u. a. an dem deutlichen Druck zu Selbstvermarktung sichtbar wird. Was aber auch deutlich wird, ist, dass die intersektionale Mehrebenenanalyse nicht nur die eine Seite sozialer Ungleichheit - Benachteiligung und Diskriminierung - sichtbar macht, sondern auch die andere, nämlich Privilegierung. Paula ist offenbar in mehrfacher Hinsicht eher privilegiert, aber gerade an ihren Privilegien wird deutlich, in welchen Kontexten des Internets und entlang welcher Problemlagen soziale Ungleichheiten entstehen und sich verfestigen, denn ihre Privilegien sind die Benachteiligungen anderer. So kann der technologische Wandel, der Paula „nur“ anstrengt und stresst, für andere, die nicht „hinterherkommen“, zum Benachteiligungsfaktor werden.

\section{Fazit}

Das skizzierte Vorgehen ist der Versuch, entlang der von Winker/Degele (2009) vorgeschlagenen intersektionalen Mehrebenenanalyse die Wechselwirkungen und Zusammenhänge von Identitätskonstruktionen, symbolischen Repräsentationen und sozialen Strukturen mit den dort wirksamen verschiedenen Differenzierungskategorien herauszuarbeiten und diese mit den verschiedenen Forschungssträngen der Internetforschung zusammenzubinden, um damit ein umfassendes Bild von sozialer Ungleichheit in $\mathrm{Zu}$ gang, Nutzung, Konstruktion und „Cyberspace“ zu ermitteln.

Für die Internetforschung ist dies insofern weiterführend, als sich die Unterscheidung in die drei Ebenen Struktur, Repräsentation und Identität als sinnvoll erwiesen hat, wird auf diese Weise beispielsweise deutlich, dass die Nutzungsaufforderungen, die die Subjekte wahrnehmen, Effekte sowohl struktureller als auch diskursiver Bedingungen sind. Die analytische Trennung von Struktur, Repräsentation und Identität und die damit verbundene Perspektive auf die Wechselwirkungen zwischen ihnen bietet zudem insbesondere im Feld der Internetforschung insofern Potenziale für neue Einsichten, als über diese Perspektive auch das spannungsreiche Verhältnis zwischen Konstruktionsweisen, d. h. den zu Handlungen auffordernden Artefakten, und den mehr oder weniger eigensinnigen Nutzungsweisen neu betrachtet werden kann, nicht zuletzt, wenn wie vorgeschlagen mit verschiedenen Methoden gearbeitet wird. Fragen nach Nutzungs- und Konstruktionsweisen, die bisher oft getrennt verhandelt werden, können dann gemeinsam analysiert werden. Das gleiche gilt für die Verschränkung von Nutzungsweisen und virtuellen Identitäten im „Cyberspace“, auch wenn dies hier nicht durch Beispiele illustriert werden konnte. 
Auch sollte deutlich geworden sein, welche Vorteile es hat, die Auswertung zunächst an der Perspektive der Subjekte zu orientieren. Statt gleich zu Beginn von außen angelegte Diskriminierungsmerkmale und Ungleichheiten im Kontext des Internets an die Subjekte heranzutragen, stehen subjektive Sichtweisen - und über diese auch eine Verbindung zwischen Zugang, Nutzung, Artefakten und „Cyberspace“ - im Zentrum. Auf diese Weise können neue Dimensionen sozialer Ungleichheit identifiziert werden, die über bekannte Faktoren wie Medienkompetenz, Motive oder Interessen hinausgehen. In Fall unseres Beispiels kristallisierte sich dabei der Umgang mit dem beschleunigten technologischen Wandel als Dimension heraus. Auch der Fokus auf konkrete Praxen und Situationen ermöglicht differenzierte Analysen von Zugang, Nutzung, Konstruktionsweisen oder virtuellen Räumen, da an diesen Praxen beispielsweise sichtbar wird, wie voraussetzungsreich, ausgrenzend oder unterstützend das Internet ist und wie die Subjekte darauf reagieren.

Die intersektionale Mehrebenenanalyse wiederum gewinnt durch den Transfer in die Internetforschung wichtige Erkenntnisse über die für Praxen konstitutiven technischmedialen Artefakte hinzu. Insbesondere auf der Strukturebene gelangen damit neben Organisationen und Institutionen auch materiell-technisch-mediale Strukturen mit ihrem handlungsleitenden bzw. auffordernden Charakter in den Fokus.

Durch die Kombination der Methoden sozialwissenschaftlicher Internetforschung und der intersektionalen Mehrebenenanalyse kann es somit gelingen, die diversifizierte Forschung zu sozialer Ungleichheit im Kontext des Internets zusammenzudenken und damit auch neue Erkenntnisse über die Konstitutionsprozesse sozialer Ungleichheiten zu gewinnen.

\section{Literatur}

Ahrens, Julia (2009): Going online, doing gender. Alltagspraktiken rund um das Internet in Deutschland und Australien. Bielefeld: Transcript.

Albrecht, Gary L. (2006) (Hg.): Encyclopedia of Disability. Thousand Oaks: Sage.

Barlow, John Perry (1996): Unabhängigkeitserklärung des Cyberspace. In: Bollmann, Stefan/Heibach, Christine (Hg.): Kursbuch Internet. Anschlüsse an Wirtschaft und Politik, Wissenschaft und Kultur. Mannheim: Bollmann, 110-118.

Becker, Barbara/Paetau, Michael (Hg.) (1997): Virtualisierung des Sozialen. Die Informationsgesellschaft zwischen Fragmentierung und Globalisierung. Frankfurt am Main/New York: Campus.

Brah, Avtar/Phoenix, Ann (2004): „Ain’t I A Woman? Revisiting Intersectionality“. In: Journal of International Women's Studies, Vol. 5, No. 3, 75-86.

Bühl, Achim (1998): Herrschaftsfrei und grenzenlos? Eine Politische Soziologie des Internet. In: Imbusch, Peter (Hg.): Macht und Herrschaft. Sozialwissenschaftliche Konzeptionen und Theorien. Opladen: Leske+Budrich, 353-370.

Bührmann, Andrea D. (2009): Intersectionality - ein Forschungsfeld auf dem Weg zum Paradigma? Tendenzen, Herausforderungen und Perspektiven der Forschung über Intersektionalität. In: Gender. Zeitschrift für Geschlecht, Kultur und Gesellschaft, Jg. 1, Nr. 2, 28-44.

Busemann, Katrin/Gscheidle, Christoph (2010): Web 2.0: Nutzung steigt - Interesse an aktiver Teilhabe sinkt. In: Media Perspektiven, Nr. 7-8, 359-368.

Butler, Judith (1991): Das Unbehagen der Geschlechter. Frankfurt/M.: Suhrkamp.

Carby, Hazel V. (1982): „White Women Listen! Black Feminism and the Boundaries of Sisterhood“. In: The Centre for Contemporary Culture Studies (Ed.): The Empire Strikes Back. Race and Racism in 70s Britain. London: Routledge, 212-235.

Carstensen, Tanja (2007): Die interpretative Herstellung des Internet. Eine empirische Analyse technikbezogener Deutungsmuster am Beispiel gewerkschaftspolitischer Diskurse. Bielefeld: Kleine Verlag. 
Carstensen, Tanja (2008): Zur Ko-Konstruktion von Technik und Geschlecht in Diskursen über das Internet. In: Alumni-Verein Hamburger Soziologinnen und Soziologen e. V. (Hg.): Lebendige Soziologie. Jahrbuch 2006/2007. Hamburg, 24-41.

Carstensen, Tanja (2009): Gender Trouble in Web 2.0: Gender Relations in Social Network Sites, Wikis and Weblogs. In: International Journal of Gender, Science and Technology, Vol. 1, No. 1, http://genderandset.open.ac.uk/index.php/genderandset/article/view/18 [21.12.2011].

Carstensen, Tanja/Winker, Gabriele (2005a): A Tool but Not a Medium - Practical Use of the Internet in the Women's Movement. In: Archibald, Jacqueline/Emms, Judy/Grundy, Frances, Payne, Janet/Turner, Eva (Eds.): The Gender Politics of ICT. Middlesex, 149-162.

Carstensen, Tanja/Winker, Gabriele (2005b): Problemorientierte Suchstrategien und die Auffindbarkeit frauenpolitischer Inhalte im Internet. In: Schachtner, Christina/Winker, Gabriele (Hg.): Virtuelle Räume - neue Öffentlichkeiten. Frauennetze im Internet. Frankfurt/New York, 91-106.

Collins, Patricia Hill (1993): Toward a New Vision: Race, Class and Gender as Categories of Analysis and Connection. In: Race, Sex \& Class, Vol. 1, No. 1, 25-45.

Combahee River Collective (1982): A Black Feminist Statement. In: Hull, Gloria T./Scott, Patricia Bell/Smith, Barbara (Eds.): But Some of Us Are Brave. Black Women's Studies. Old Westbury/ New York: Feminist Press, 13-22 (Orig. von 1977).

Consalvo, Mia/Paasonen, Susanna (Hg.) (2002): Women \& Everyday Uses of the Internet. Agency $\&$ Identity. New York u. a.: Peter Lang.

Crenshaw, Kimberlé (1989): Demarginalizing the Intersection of Race and Sex: A Black Feminist Critique of Antidiscrimination Doctrine. In: The University of Chicago Legal Forum, 139-167.

Davis, Kathy (2008): Intersectionality as Buzzword. A Sociology of Science Perspective on what makes a Feminist Theory successful. In: Feminist Theory, Vol. 9, No. 1, 67-85.

DiMaggio, Paul/E. Hargittai, Eszter (2001): From the 'Digital Divide' to 'Digital inequality'. Studying Internet Use as Penetration Increases. Princeton University Center for Arts and Cultural Policy Studies, Working Paper Series number 15.

Dobransky, Kerry/Hargittai, Eszter (2006): The Disability Divide in Internet Access and Use. In: Information, Communication and Society, Vol. 9, No. 3, 313-334.

Emmer, Martin/Füting, Angelika/Vowe, Gerhard (2006): Wer kommuniziert wie über politische Themen? Eine empirisch basierte Typologie individueller politischer Kommunikation. In: Medien \& Kommunikationswissenschaft Jg. 54, Nr. 2, 216-236.

Fairlie, Robert W. (2004): Race and the Digital Divide. In: Contributions to Economic Analysis \& Policy 3, 1, Article 15, http://www.bepress.com/bejeap/contributions/vol3/iss1/art15 [21.12.2011].

Fuglsang, Lars (2005): IT and Senior Citizens. Using the Internet for Empowering Active Citizenship. In: Science, Technology \& Human Values, Vol. 30, No. 4, 468-495.

Gerhards, Maria/Mende, Annette (2009): Offliner: Ab 60-jährige Frauen bilden die Kerngruppe. In: Media Perspektiven, Nr. 7, 365-376.

Gerhards, Maria/Klinger, Walter/Trump, Thilo (2008): Das Social Web aus Rezipientensicht: Motivation, Nutzung und Nutzertypen, in: Zerfaß, Ansgar/Welker, Martin/Schmidt, Jan (Hg.): Kommunikation, Partizipation und Wirkungen im Social Web. Grundlagen und Methoden: Von der Gesellschaft zum Individuum. Köln: Halem, 129-148.

Groß, Melanie/Carstensen, Tanja/Winker, Gabriele (2008): Wider das Überflüssig-sein. Kompensationsversuche erwerbsloser Personen, http://www.tu-harburg.de/agentec/publikationen/ue berfluessig_2008.pdf [21.12.2011].

Hacker, Hanna (2007): Norden. Süden. Cyberspace. Text und Technik gegen die Ungleichheit, Wien: Promedia.

Harding, Sandra (1991): Feministische Wissenschaftstheorie. Zum Verhältnis von Wissenschaft und sozialem Geschlecht. Hamburg: Argument.

Hargittai, Eszter (2002): Second-Level Digital Divide. Differences in People's Online Skills. In: First Monday, 7, 4, http://chnm.gmu.edu/digitalhistory/links/pdf/introduction/0.26c.pdf [21.12.2011].

Hark, Sabine (1993): Queer Intervention. In: Feministische Studien, Kritik der Kategorie „Geschlecht“, Jg. 11, No. 2, 103-109. 
Henning, Florian (2008): „Ich glaube nicht an Grenzen“. Virtuelle Öffentlichkeiten türkischstämmiger Jugendlicher in den Niederlanden. In: Wischermann, Ulla/Thomas, Tanja ( $\mathrm{Hg})$ : Medien - Diversität - Ungleichheit. Wiesbaden: VS Verlag für Sozialwissenschaften, 183-202.

Hepp, Andreas (2009): Digitale Medien, Migration und Diaspora. Deterritoriale Vergemeinschaftung jenseits nationaler Integration. In: Hunger, Uwe/Kissau, Kathrin (Hg.): Internet und Migration. Wiesbaden: VS Verlag für Sozialwissenschaften, 35-54.

Hepp, Andreas/Suna, Laura/Welling, Stefan (2009): Integrations- und Segregationspotenziale: Digitale Medien und Migration. In: Ulbrich, Hans-Joachim/Hoffmann, Bernward (Hg.): Geteilter Bildschirm - Getrennte Welten? Konzepte für Pädagogik und Bildung. München: Kopäd, 89-98.

Hesse, Franka (2008): Die Geschlechterdimension von Weblogs: Inhaltsanalytische Streifzüge durch die Blogosphäre. In: kommunikation@gesellschaft, Jg. 9, http://www.soz.uni-frank furt.de/K.G/B1_2008_Hesse.pdf [21.12.2011].

Hörning, Karl H. (2001): Experten des Alltags. Die Wiederentdeckung des praktischen Wissens. Weilerswist: Velbrück Wissenschaft.

Hörning, Karl H./Reuter, Julia (2004): Doing Culture: Kultur als Praxis. In: Hörning, Karl H./ Reuter, Julia (Hg.): Doing Culture. Neue Positionen zum Verhältnis von Kultur und sozialer Praxis. Bielefeld: Transcript, 9-15.

Hunger, Uwe/Kissau, Kathrin (Hrsg.) (2009): Internet und Migration. Wiesbaden: VS Verlag für Sozialwissenschaften.

Hurrelmann, Bettina (2002): Zur historischen und kulturellen Relativität des „gesellschaftlich handlungsfähigen Subjekts“ als normativer Rahmenidee für Medienkompetenz. In: Groeben, Norbert/dies. (Hg.): Medienkompetenz. Weinheim/München: Juventa, 111-126.

Initiative D21 (2011): (N)Onliner-Atlas 2011 - eine Topographie des digitalen Grabens durch Deutschland. Nutzung und Nichtnutzung des Internets, Strukturen und regionale Verteilung. http://www.initiatived21.de/wp-content/uploads/2011/07/NOnliner2011.pdf [21.12.2011].

Iske, Stefan/Klein, Alexandra/Kutscher, Nadia (2004): Nutzungsdifferenzen als Indikator für soziale Ungleichheit im Internet. In: kommunikation@gesellschaft, Jg. 5, http://www.soz.unifrankfurt.de/K.G/B3_2004_Iske_Klein_Kutscher.pdf [21.12.2011].

Jäckel, Michael/Lenz, Thomas/Zillien, Nicole (2005): Die regionale digitale Spaltung. Eine empirische Studie zu Unterschieden in der Internetnutzung in Stadt und Land. Trier: ceb-Schriftenreihe zum E-Business.

Jacob, Jutta/Köbsell, Swantje/Wollrad, Eske (Hg.) (2010): Gendering Disability. Intersektionale Aspekte von Behinderung und Geschlecht. Bielefeld: Transcript.

Kelle, Udo/Kluge, Susanne (1999): Vom Einzelfall zum Typus. Opladen: Leske+Budrich.

Kleinsteuber, Hans J. (1995): Technologies of Freedom - Warum werden in den USA Medien so ganz anders interpretiert? In: Amerikastudien/American Studies, Jg. 40, Nr. 2, 183-208.

Knapp, Gudrun-Axeli (2005): „Intersectionality“ - ein neues Paradigma feministischer Theorie? In: Feministische Studien, Jg. 23, Nr. 1, 68-81.

Krotz, Friedrich (2001): Mediatisierung von Alltag, Kultur und Gesellschaft. Ein gesellschaftlicher Metaprozess wird besichtigt. Wiesbaden: VS Verlag für Sozialwissenschaften.

Kubicek, Herbert/Dutton, William H./Williams, Robin (Eds.) (1997): The Social Shaping of Information Superhighways. European and American Roads to the Information Society. Frankfurt/New York: Campus.

Leggewie, Claus/Maar, Christa (Hg.) (1998): Internet und Politik. Von der Zuschauer- zur Beteiligungsdemokratie. Köln: Bollmann.

Lenhart, Amanda/Madden, Mary (2007): Teens, Privacy \& Online Social Networks. How Teens Manage their Online Identities and Personal Information in the Age of MySpace. In: PEW Internet \& American Life Project, Washington D.C., http://www.pewinternet.org/ /media// Files/Reports/2007/PIP_Teens_Privacy_SNS_Report_Final.pdf.pdf [21.12.2011].

Lutz, Helma/Wenning, Norbert (2001): „Differenzen über Differenz - Einführung in die Debatten“. In: Lutz, Helma/Wenning, Lutz (Hg.): Unterschiedlich verschieden. Differenz in der Erziehungswissenschaft. Opladen: Leske+Budrich, 11-24. 
Manago, Adriana M./Graham, Michael B./Greenfield, Patricia M./Salimkhan, Goldie (2008): SelfPresentation and Gender on MySpace. In: Journal of Applied Developmental Psychology, Vol. 29, No. 6, 446-458.

Marr, Mirko (2005): Internetzugang und politische Informiertheit. Zur digitalen Spaltung der Gesellschaft. Konstanz: UVK.

Mocigemba, Dennis (2006): Warum sie selber senden: Eine Typologie von Sendemodi im Podcasting. In: kommunikation@gesellschaft, Jg. 7, http://www.soz.uni-frankfurt.de/K.G/ B3_2006_Mocigemba.pdf [21.12.2011].

Mossberger, Karen/Tolbert, Caroline J./Stansbury, Mary (2003): Virtual Inequality. Beyond the Digital Divide. Washington, DC: Georgetown University Press.

Münker, Stefan (2009): Emergenz digitaler Öffentlichkeiten. Die Sozialen Medien im Web 2.0. Frankfurt am Main: Suhrkamp.

Münker, Stefan/Roesler, Alexander (Hg.) (2002): Praxis Internet. Frankfurt/M.: Suhrkamp.

Neverla, Irene (1998): Das Medium denken. Zur sozialen Konstruktion des Netz-Mediums. In.: dies. (Hg.): Das Netz-Medium. Kommunikationswissenschaftliche Aspekte eines Mediums in Entwicklung. Opladen/Wiesbaden: Westdeutscher Verlag, 17-35.

Norris, Pippa (2001): Digital Divide. Civic Engagement, Information Poverty, and the Internet Worldwide. Cambridge: Cambridge University Press.

Oliver, Michael (1996): Understanding Disability: From Theory to Practice. New York: St. Martin’s Press.

Ono, Hiroshi (2006): Digital Inequality in East Asia: Evidence from Japan, South Korea and Singapore. In: Asian Economic Papers, Vol. 4, No. 3, 116-39.

Paulitz, Tanja (2005): Netzsubjektivität/en. Konstruktionen von Vernetzung als Technologien des sozialen Selbst. Eine empirische Untersuchung in Modellprojekten der Informatik. Münster: Westfälisches Dampfboot.

Pinch, Trevor/Wiebe Bijker (1987): The Social Construction of Facts and Artifacts: Or How the Sociology of Science and the Sociology of Technology Might Benefit Each Other. In: Bijker, Wiebe/Hughes, Thomas/Pinch, Trevor (Eds.): The Social Construction of Technological Systems. New Directions in the Sociology and History of Technology. Cambridge, Mass.: MIT Press, 17-50.

Reckwitz, Andreas (2003): Grundelemente einer Theorie sozialer Praktiken. Eine sozialtheoretische Perspektive. In: Zeitschrift für Soziologie Jg. 32, Nr. 4, 282-301.

Rheingold, Howard (1994): Virtuelle Gemeinschaften. Soziale Beziehungen im Zeitalter des Computers. Bonn: Addison-Wesley.

Rommes, Els/van Oost, Ellen/Oudshoorn, Nelly (1999): Gender and the Design of a Digital City. In: Information Technology, Communication and Society, Vol. 2, No. 4, 476-495.

Schachtner, Christina (2009): Wissen und Gender. Der Cyberspace als genderrelevanter Wissensraum. In: Medien \& Kommunikationswissenschaft, Jg. 57, Nr. 4, 500-519.

Schmidt, Jan (2006): Weblogs. Eine kommunikationssoziologische Studie, Konstanz: UVK.

Schönberger, Klaus (1999): Internet zwischen Spielwiese und Familienpost. Doing Gender in der Netznutzung. In: Hebecker, Eike/Kleemann, Frank/Neymanns, Harald/Stauff, Markus (Hg.): Neue Medienumwelten. Zwischen Regulierungspraxis und alltäglicher Aneignung. Frankfurt am Main/New York: Campus, 259-280.

Stegbauer, Christian (2001): Grenzen virtueller Gemeinschaft. Strukturen internetbasierter Kommunikationsforen. Wiesbaden: Westdeutscher Verlag.

Stegbauer, Christian (2009): Wikipedia. Das Rätsel der Kooperation. Wiesbaden: VS Verlag für Sozialwissenschaften.

Thiedeke, Udo (Hg.) (2004): Soziologie des Cyberspace. Medien, Strukturen und Semantiken. Wiesbaden: VS Verlag für Sozialwissenschaften.

Thomas, Graham/Wyatt, Sally (1999): Shaping Cyberspace - Interpreting and Transforming the Internet. In: Research Policy, Vol. 28, No. 7, 681-698.

Turkle, Sherry (1998): Leben im Netz. Identität in Zeiten des Internet. Reinbek: Rowohlt.

van Eimeren, Birgit/Frees, Beate (2011): Drei von vier Deutschen im Netz - ein Ende des digitalen Grabens in Sicht? Ergebnisse der ARD/ZDF-Onlinestudie 2011. Media Perspektiven Nr. 7-8, 334-349. 
van Oost, Ellen (2003): Materialized Gender: How Shavers Configure the Users' Femininity and Masculinity. In: Oudshoorn, Nelly E. J./Pinch, Trevor J. (Eds.): How Users Matter: The CoConstruction of Users. Cambridge, M.A.: MIT Press, 194-208.

Wasserman, Ira M./Richmond-Abbott, Marie (2005): Gender and the Internet: Causes of Variation in Access, Level, and Scope of Use. In: Social Science Quarterly, Vol. 86, No. 1, 252-70.

Wehling, Peter (2006): The Situated Materiality of Scientific Practices: Postconstructivism - a New Theoretical Perspective in Science Studies? In: Science, Technology \& Innovation Studies, Special Issue, Vol. 2, No. 1: After Constructivism in Science and Technology Studies, 81-100.

Weizenbaum, Josef (1999): Das Internet ist ein Schrotthaufen - mit Perlen drin, Interview mit Joseph Weizenbaum. In: Der Tagesspiegel, 28. Februar 1999.

Wellman, Barry/Haythornthwaite, Caroline A. (Eds.) (2002): The Internet in Everyday Life. Malden/Oxford/Carlton: Blackwell Publishing.

Wetterer, Angelika (2003): Rhetorische Modernisierung: Das Verschwinden der Ungleichheit aus dem zeitgenössischen Differenzwissen. In: Knapp, Gudrun Axeli/Wetterer, Angelika (Hg.): Achsen der Differenz. Gesellschaftstheorie und feministische Kritik II. Münster, 286-319.

Winker, Gabriele (2002): Informationstechnik und Geschlechterhierarchie - eine bewegende Beziehung. In: Technikfolgenabschätzung. Theorie und Praxis, Jg. 11, Nr. 2, Juli, 70-78.

Winker, Gabriele (2004): Internetforschung aus Genderperspektiven. In: Buchen, Sylvia/Helfferich, Nena/Maier, Maja (Hg.): Gender methodologisch. Empirische Forschung in der Informationsgesellschaft vor neuen Herausforderungen? Wiesbaden: VS Verlag für Sozialwissenschaften, 123-140.

Winker, Gabriele/Degele, Nina (2009): Intersektionalität. Zur Analyse sozialer Ungleichheiten. Bielefeld: Transcript.

Winker, Gabriele/Degele, Nina (2011): Intersectionality as Multi-Level-Analysis: Dealing with Social Inequality. In: European Journal of Women's Studies, Vol. 18, No.1, 51-66.

Winner, Langdon (1985): Do Artifacts Have Politics? In: MacKenzie, Donald/Wajcman, Judy (Eds.): The Social Shaping of Technology. How the Refrigerator Got its Hum. Milton Keynes/ Philadelphia: Open University Press, 26-38.

Wötzel-Herber, Henning (2008): Doing Me and the Others. Identitätskonstruktionen in OnlineCommunities. Unveröffentlichte Masterarbeit, Universität Hamburg, http://woetzel-her ber.de/wp-content/uploads/2009/02/doing_me_final_web.pdf [21.12.2011].

Yuval-Davis, Nira (2006): Intersectionality and Feminist Politics. In: The European Journal of Women's Studies, Vol. 13, No. 3, 193-209.

Zillien, Nicole (2006): Digitale Ungleichheit. Neue Technologien und alte Ungleichheiten in der Informations- und Wissensgesellschaft. Wiesbaden: VS Verlag für Sozialwissenschaften. 